\title{
Nacer entre la ceiba y el río: algunas prácticas de crianza ticuna*
}

\author{
Born between Ceiba and the River: Some Ticuna Parenting Practices \\ Juliet Carvajal Hernández Julián Felipe Cantor ${ }^{2}$ Maribel Reyes Reyes ${ }^{3}$
}

Para citar este artícullo: Carvajal, J.; Cantor, J. F.; Reyes, M. (2016). Nacer entre la ceiba y el río: algunas prácticas de crianza ticuna. Infancias Imágenes, 15(2), 181-193.

\section{Resumen}

El presente artículo de reflexión expone los hallazgos obtenidos en el marco de un proyecto de investigación titulado Una aproximación desde el relato mítico y el rito a las prácticas de crianza en familias indígenas Ticuna, el cual propende el reconocimiento de las distintas prácticas culturales de infancia en la comunidad Doce de octubre del Amazonas colombiano. Algunos de los resultados presentados aquí corresponden a los hallazgos en cuanto a la preparación de saberes y oficios para la constitución de familia, nacimiento, cuidados y creencias, dieta, enfermedades y rito de la pelazón, las cuales son temáticas emergentes que surgieron a partir de un acercamiento a la realidad social y cultural de la comunidad. Desde allí contribuimos al análisis lingüístico que resalta los métodos propios de la oralidad indígena, pues este pensamiento mitológico es transmitido oralmente para la perpetuación de su cultura.

Palabras clave: lenguaje hablado, conocimientos tradicionales, crianza del niño, nacimiento, Amazonía [UNESCO Thesaurus http://vocabularies.unesco.org/browser/thesaurus/es/]
Recibido: 30-abril-2016 / Aprobado: 27-julio-2016

\begin{abstract}
This reflection paper exposes the findings obtained in the framework of a research project entitled "An approach from mythical narrative and ritual to rearing practices in Ticuna Indian families", which promotes recognition of different cultural practices of children at the Doce de octubre community in the Colombian Amazon region. Some of the results presented here correspond to findings regarding the preparation of knowledge and crafts for the formation of family, birth, care and beliefs, diet, diseases and rite of hair cutting, which are emerging topics that arose from an approach to the social and cultural realities of the community. From there, we contribute to linguistic analysis that highlights the methods of indigenous oral tradition, as this mythological thinking is transmitted orally for the perpetuation of their culture.
\end{abstract}

Keywords: Spoken language, Traditional knowledge, Child rearing, Birth, Amazonia.

\footnotetext{
* Artículo de revisión derivado de la investigación denominada "Una aproximación desde el relato mítico y el rito a las prácticas de crianza en familias indígenas Ticuna" desde el año 2014 hasta abril de 2015 en la comunidad Doce de Octubre, en Amazonas (Colombia), para optar al título de especialista en Infancia, Cultura y Desarrollo de la Universidad Distrital Francisco José de Caldas.

1 Licenciada en Psicología y Pedagogía. Especialista en Infancia Cultura y Desarrollo, Universidad Distrital Francisco José de Caldas. Asistente académico, IEIE, Universidad Distrital Francisco José de Caldas. Correo electrónico: julcarvajal@ hotmail.com.

2 Licenciado en Educación Básica con énfasis en Ciencias Sociales. Especialista en Infancia Cultura y Desarrollo, Universidad Distrital Francisco José de Caldas. Coordinador operativo, Internacional compañía de financiamiento. Correo electrónico: juliancantor92@gmail.com.

3 Licenciada en Educación Básica con énfasis en Educación Artística. Especialista en Infancia Cultura y Desarrollo, Universidad Distrital Francisco José de Caldas. Artista formadora de danzas, Idartes. Correo electrónico: maribelreyes12@hotmail.com.
} 


\section{Introducción}

La Especialización en Infancia, Cultura y Desarrollo de la Universidad Distrital Francisco José de Caldas fue el escenario de encuentro de un grupo de investigadores interesados en la infancia. Las discusiones derivadas de las clases fueron cruciales para interrogar los procesos dados en la familia respecto a la infancia en contextos diferentes a la ciudad; esto condujo a la búsqueda de realidades culturales de los territorios indígenas en nuestro país. Se presenta entonces, un trabajo investigativo que plantea una tensión entre la perspectiva occidental y perspectivas otras frente a la infancia, el cual fue pertinente porque logró ampliar las discusiones y análisis que se llevan al interior de la academia, dando como resultado, por un lado, un reconocimiento pluricultural a las prácticas de crianza y, por otro, complejizar las posturas epistémicas que fundan la mirada sobre la infancia en el marco institucional.

En Colombia, el departamento de Amazonas es habitado en su gran mayoría por pueblos indígenas y

ha manifestado históricamente una resistencia cultural para la preservación de su alteridad en el mundo occidental. En la actualidad, existe, a la ribera del río Loretoyacu, la comunidad ticuna Doce de octubre, quienes posibilitaron una indagación de corte etnográfico, es decir, a través la inmersión en su cotidianidad. Allí se brindó un entramado de vivencias, narraciones y costumbres como hilos trenzados para comprender las interacciones culturales de la comunidad que aportarían a un acercamiento a las prácticas de crianza al interior de las familias ticuna.

\section{Marco teórico}

En el desarrollo de la investigación emergieron de manera categórica algunas nociones conceptuales que ubicaron y orientaron las rutas para ahondar en la cultura indígena ticuna. A continuación, se mostrará brevemente algunos postulados, premisas y estudios realizados en el campo por académicos como Jean Pierre Goulard, entre otros, que orientaron la indagación sobre las prácticas de crianza en familias ticuna.

\section{Comunidad Doce de octubre, un legado del pueblo ticuna}

La comunidad indígena Doce de octubre, ubicada a la ribera del río Loretoyacu que lindera con Perú, según el censo poblacional (2015), se constituye por 323 habitantes organizados en 64 familias que en su gran mayoría pertenecen a la etnia ticuna, con una organización social definida por clanes o nazones de parentesco. Esta comunidad se encuentra a $40 \mathrm{~km}$ en embarcación de la cabecera municipal de Puerto Nariño, donde las comunidades pueden acceder a servicios del Estado como lo son: salud, educación de carácter religioso en un internado para estudiantes de bachillerato y funciones ciudadanas, que usualmente son trámites de proyectos sociales o el ejercicio de votación.

El Doce de octubre pertenece a la jurisdicción del resguardo Aticoya, es decir, hace parte de la asociación indígena Tikunas, Cocamas y Yaguas, donde se toman decisiones políticas, como son las concertación y participación en asambleas departamentales y participación política en disertaciones sobre los proyectos gubernamentales sociales de gobierno nacional para implementar en el territorio.

La organización política es derivada del plan de vida, documento que constituye leyes y normas para una representación política como pueblo indígena ante la Constitución Política. Allí se presentan diferentes actividades competentes al orden social; los representantes o líderes comunitarios contribuyen al desarrollo respecto a las necesidades de administración, educación, salubridad, espiritualidad y logística que requieren los pobladores. Por consiguiente, la figura representativa de las decisiones en comunidad es el curaca, elegido por votación, y su comité de trabajo conformado por el promotor de salud, guardia indígena, etnoeducadores, abuelos y parteras, quienes responden a los intereses primarios que le sean solicitados.

\section{Entre la modernidad y lo tradicional}

\footnotetext{
Hoy día los niños tienen que estudiar, si un niño se queda por ahí pues no va hacer nada.

$Y$ para eso vienen los profesores,

las monjas y mucha gente a enseñar,

entonces nosotros tenemos que demostrar nuestras capacidades,

porque también nosotros podemos tener un trabajo, podemos manejar un computador.
}

Mark Arcesio Ahué, indígena ticuna 
La cultura ticuna anida sus costumbres en concepciones mitológicas que orientan las maneras aceptadas de ser en colectivo y condicionan su manera de habitar, sentir y ser en un territorio dotado de redes de sentido. Para entender los entramados de cultura y las formas que adopta de acuerdo al tiempo y espacio en el que se mueve los postulados de Néstor Canclini (2012) señalaron una lectura minuciosa sobre las dinámicas propias de la cultura y las dificultades de observar, analizar una cultura pura, mostrando además cómo las intenciones geopolíticas han influido en este fenómeno.

Un concepto central del autor para la comprensión de la cultura en el contexto latinoamericano fue el de culturas híbridas. En palabras del autor, la cultura transita de manera simultánea con diversas estructuras de pensamiento, creando así mixturas que construyen nuevas formas de identidad latinoamericana. Una posible causa es la distribución territorial globalizada, que causa relaciones geopolíticas que segregan las otras culturas, creando síntomas como la desigualdad y desconexión entre las sociedades que superviven desde diferentes lógicas de pensamiento, prácticas y tiempos culturales. Es así como la fusión entre modernidad y lo tradicional reorganiza una pluralidad de sentidos compartidos (Canclini, 2012).

La perspectiva cultural expuesta por (Canclini, 1998) advierte que la idea de civilización fue implantada para la transnacionalización de una única cultura superior sobre otras inferiores. Comprender que las relaciones económicas y políticas, naturalizan prácticas culturales en la vida cotidiana, complejizando los modos de convivir de diversas lenguas y estilos de vida conectados por una intensión económica transnacional. Con lo dicho anteriormente, el autor explica cómo la cultura dada en tiempos de globalización "no se ve como comunicación entre los diferentes, sino como una expansión de las culturas hegemónicas y de versiones estilizadas de la otredad" (Canclini, p. 2, 2012). Además, advierte cómo las culturas reconocidas como inferiores han sido desacralizadas por la entrada del pensamiento científico occidental, sobre los pueblos indígenas subyugados para su producción, desacreditando el pensamiento mítico y los rituales de las sociedades denominadas primitivas, adjetivo reiterativo en las representaciones del otro por su particularidad indígena, idea implantada y aceptada históricamente en la cultura.

\section{Sentido mítico en la cultura indígena ticuna}

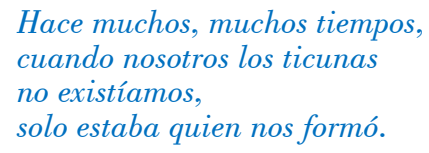

Edgar Valentín, joven ticuna

Entender la función del mito en la estructura del pensamiento cultural indígena fue posible a través de la lectura minuciosas de autores como Jon Landaburu (1984), quien explica cómo el mito resulta ser una construcción ordenada y sistemática del mundo que da sentido a las prácticas y/o rituales para sostener una memoria ancestral latente en la oralidad y que, si bien no es antecesor del pensamiento científico ni superior, coexisten de manera paralela. Dicho autor señala cómo “...de punta a punta el mito es un pensamiento simbólico, una ordenación dialéctica del mundo" (Landaburu, p. $56,1984)$, en donde los personajes que emergen en la narración están dotados de diferentes connotaciones que develan el viraje de la realidad hacia la constitución de una narrativa polivalente en significados. De tal manera, los mitos son un constructo de conocimiento al interior de las comunidades indígenas y es constituido desde la oralidad para estar vivo en sus ritos.

Según Goulard (2009), el sentido mítico en la cultura ticuna y en la constitución del ser en esta comunidad instaura un orden en la forma de vivir y relacionarse con los otros, además, configura la presencia del tiempo como acto de recordar, que al hacer memoria dibuja la historia. Por lo anterior, y en lo evidenciado en la investigación, la oralidad es el mecanismo para rememorar el origen. En este sentido, el tiempo es una categoría de la oralidad en la cultura ticuna que organiza sus creencias donde, según el autor, "relatan la historia de la cotidianidad, para situar su experiencia dentro de una temporalidad cosmogónica partiendo del mito de origen y de allí referencian el presente y su proximidad al tiempo de los antiguos" (p 37, 2009). Adicionalmente, 
explica que para los ticuna el mundo anterior fue la causa del presente, transformando los estados materiales y conservando la esencia, de ahí que "la vida tome distintas formas: humanas y no humanas para habitar este mundo" (p. 19, 2009).

Por lo anterior, se entiende que las culturas orales conservan su sentido mítico de origen como una red de significaciones vividas en el relato de su diario vivir unidos a la narración histórica. En la oralidad, el mito recrea la realidad al ofrecer variables novedosas que son admitidas socialmente y que constituye la versión válida. La historia entonces es una interpretación sobre el tiempo que vincula el pasado y presente en una misma narración. Por esto la tradición oral mantiene el mito otorgándole un significado que explica el origen de la creación cimentado como verdad en la cultura.

\section{Oralidad: hacia una metáfora del mundo}

Nosotros las creencias de nosotros dicen que hay veces viene un espíritu malo y le acaricia al niño.

Aliria Suárez, madre ticuna

La oralidad es fundamental para llevar a cabo los rituales y prácticas en el territorio. Es por esto que la lengua materna es el código lingüístico por excelencia, en el cual están contenidas las significaciones simbólicas y representaciones psíquicas del mundo. Como lo señala el lingüista Evelio Cabrejo (2007, p.75), la oralidad traza y construye redes de sentido al mundo; desde temprana edad la madre interactúa por medio de la palabra, es así que transmite la herencia cultural.

En la comunidad ticuna la palabra tiene un sentido mucho mayor ya que con ella se transmiten los conocimientos heredados de generaciones enteras, es el canal de comunicación para la formación personal, cultural y social de la comunidad. Los abuelos son figuras en la comunidad portadoras de saberes. Su experiencia de vida los ha convertido en relatores de su origen como pueblo indígena los cuales, a partir de sus narraciones y cosmología de vida, forman un criterio espiritual para convivir en el territorio.
Cabrejo (2007) enuncia cómo a través de cantos, arrullos y cuentos se constituye la lengua cotidiana en la cual se aprende a escuchar, actividad silenciosa que genera pensamiento. Es así que desde el vientre de la madre el niño comienza a ser protagonista de lo que oye y a partir de allí interactúa con el mundo. La oralidad permite a los integrantes de la comunidad identificarse, nombrarse y, sobre todo, sentirse únicos ya que su lengua nativa les permite nombrar las cosas que los rodean y las que no y a partir de ese reconocimiento adquirir nuevas formas de concebir el mundo.

La metáfora y la alegoría, figuras propias del lenguaje, están presentes en la oralidad ticuna y permiten que estos apropien, interpreten, transformen y recreen los acontecimientos de su vida diaria para darles un sentido simbólico en el entramado cultural. Por ejemplo, como lo enuncia una abuela de la comunidad: "Es ella quien entierra la placenta, pero no muy hondo porque entre más hondo la entierra aja, así serán las raíces de los dientes". En lo anterior, se puede observar cómo en el nacimiento la placenta, al momento de ser enterrada, representa un acto simbólico que expresa una característica de sus concepciones cosmogónicas, en donde la placenta es un símbolo de unidad que influye en la vida del recién nacido. Así mismo, al relacionar el entierro de la placenta con el largo de la raíz de los dientes, expresa una metáfora, relación que para algunos no tendría ningún sentido, pero para la comunidad es un ritual que evidencia una conexión entre el ser y la naturaleza.

\section{Metodología}

Las disertaciones propuestas desde el paradigma de la investigación cualitativa son el marco desde el cual se desarrolló la investigación, con el fin de indagar las relaciones de significado al interior de una práctica cultural: prácticas de crianza en comunidades indígenas. De allí que el interés fue interpretar constructos semánticos y analizar constructos lingüísticos recopilados por medio de entrevistas semiestructuradas y el acompañamiento a sus prácticas cotidianas, con el fin de develar los significados de las acciones naturales durante la crianza.

Los principios metodológicos fueron orientados mediante un acercamiento teórico, por un lado, 
a los postulados de la antropóloga (Guber 2001), quien propone que la inmersión sea un acontecimiento natural y no forzoso en el cual los actores sociales puedan manifestar su cultura de manera habitual y el investigador se haga partícipe de su cultura desde una observación participante y, por otro, de (Garfinkel 2006), el cual estudia las situaciones de interacción, donde los actores tienen un lugar de enunciación activo como constructores de sociedad al reinterpretar sus realidades y otorgarle sentido a su contexto haciendo uso del lenguaje para compartir significados comunes, posibilitando que las narraciones orales de las comunidades sean comprendidas como métodos propios para crear conocimiento y cultura. Las fases metodológicas por las cuales se encaminó el proceso fueron: planeación, exploración, análisis e interpretación de relatos. A continuación, se describen brevemente las herramientas e instrumentos principales de indagación que posibilitaron un acercamiento a las prácticas de crianza ticuna.

\section{Recopilación de relatos}

La búsqueda se basa en una verdad narrativa que la gente construye a partir de lo que sabe de su entorno, con el fin de otorgarle significado y sentido a las acciones y rituales que se realizan cotidianamente. Así pues, "No sólo en lo que hace la gente, sino también lo que dicen que hacen, y lo que dicen que los llevó a hacer lo que hicieron, también lo que la gente dice que han hecho los otros y por qué. Y, por encima de todo, se ocupa de cómo dice la gente que es su mundo" (Bruner, p. 31, 1991).

Los relatos seleccionados fueron autobiográficos, narraciones anecdóticas de interacción con los otros en donde la verdad es construida a partir de recuerdos afectivos de lo que se piensa, siente y cree; en consecuencia, es susceptible a diferentes interpretaciones que reconstruyen la experiencia de las realidades narradas.

Esta herramienta fue clave para la investigación, pues posibilitó conocer por medio de la palabra de las madres y pobladores lo que sabían y conocían sobre la crianza y todo lo que se teje a su alrededor. A partir de esos relatos se logró construir una matriz de análisis del lenguaje donde se hallaron unidades y signos característicos de la cosmovisión indígena.

\section{Grabación y transcripción}

Según Mondada (2006), las grabaciones son fuente de análisis que arrojan expresiones, emociones y signos que permiten al investigador interpretar lo que se dice, lo que hace y cómo actúa la persona. De allí se obtiene información tomada de primera mano. La transcripción es el paso siguiente que adquieren las palabras y les otorga una forma física en oraciones y párrafos, donde se puede analizar con más detalle el discurso, después de ser transcritas sin alterar la forma en la cual se expresan las personas, destacando la riqueza verbal, las características fundamentales del tiempo en el que se realiza y el lugar en el cual se desarrolla la acción.

\section{Hallazgos}

A continuación, se presentan fragmentos de los relatos orales de madres y abuelas que fueron grabados y posteriormente transcritos para conocer y analizar lo aspectos relacionados con la crianza y los rituales que hacen parte de la cotidianidad familiar en la comunidad Doce de octubre. Las temáticas emergentes son: Preparación de saberes y oficios para la constitución de familia; Nacimiento, cuidados y creencias; Dieta y enfermedades; y Mito y rito: la pelazón. Allí se exponen los relatos organizados por unidades de sentido, generando un análisis de tipo léxico-semántico que nos acercó a la comprensión de las prácticas de crianza ticuna:

Preparación de saberes y oficios para la constitución de familia

\footnotetext{
]"Hija usted tiene que lavar los platos".

Mi padre me decía: "usted tiene que tostar fariña",

"usted tiene que ayudar a su mamá",

"tienes que aprender, ¿para qué vas a tener marido si vas a ser perezosa?"
}

Filda Gómez Ahué, madre ticuna

El padre ejerce una autoridad que impone sobre la hija para construir su lugar como mujer. Como se puede apreciar en los siguientes enunciados: "Mi padre me decía: "usted tiene que tostar fariña», «hija usted tiene que lavar los platos», «usted tiene que ayudar a su mamá» «tienes que aprender»". 


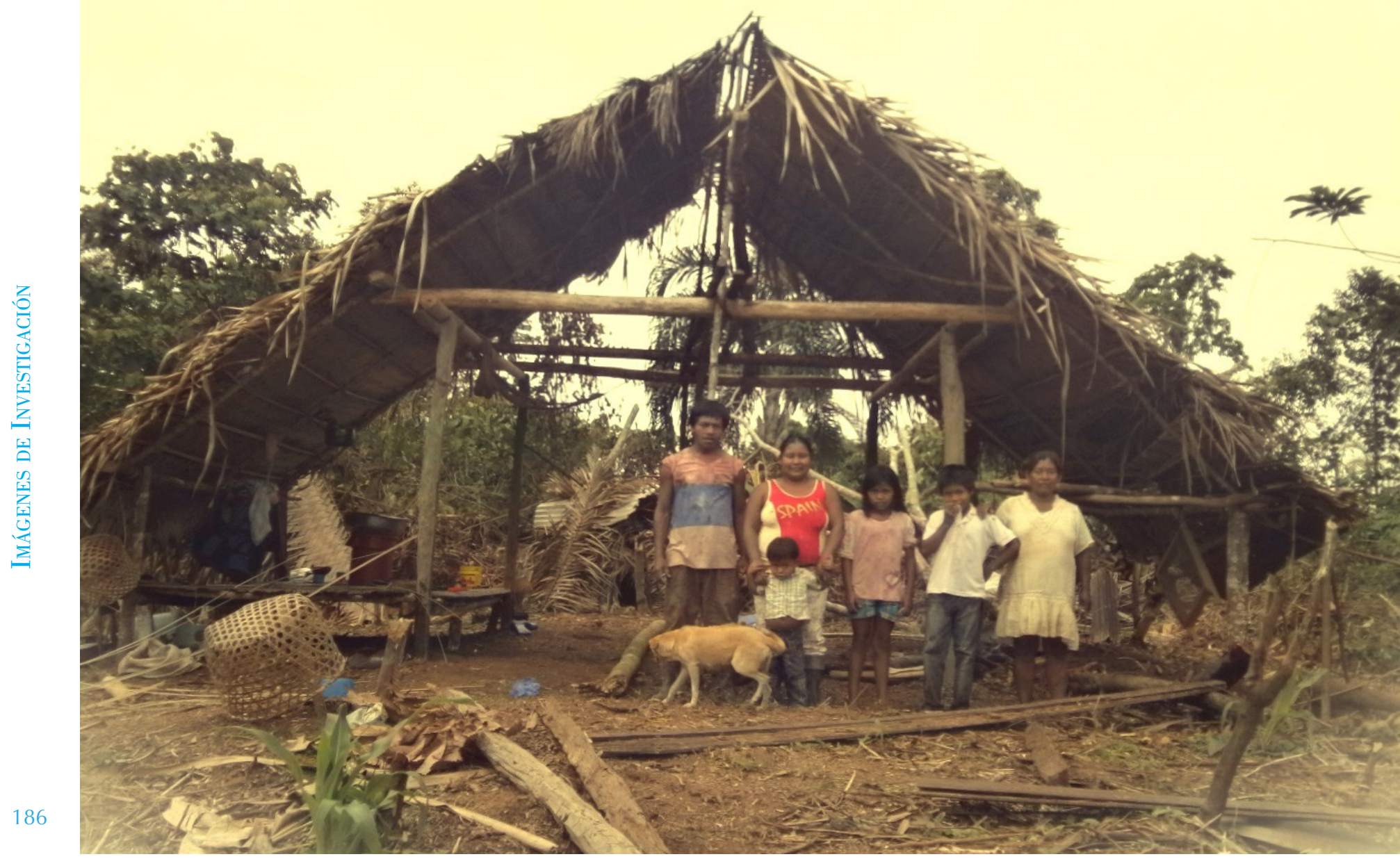

Figura 1. Familia indígena Valentín Ahué, quienes compartieron sus relatos para la presente investigación.

Fuente: archivo fotográfico de la investigación.

En la expresión del verbo en segunda persona "tie-

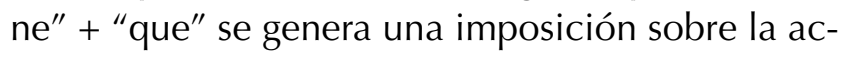
ción que se debe ejecutar; además, la orden que da el padre está enfocada en los oficios que la hija debe hacer en los diferentes espacios que habita. Incluso la expresión "usted debe ayudar a su mamá" indica el deber como hija a cumplir con los oficios y labores concernientes al trabajo del hogar, además de ser un deber pasa a ser una obligación e incita a reproducir miméticamente las mismas labores que ella.

Pues como yo desde chiquita mi mamá me crio como hacer oficio en la casa

"usted va ser grande usted desde chiquita"

Filda Gómez Ahue, madre ticuna

La relación entre madre e hija en la crianza se soporta en el aprendizaje y no aparecen expresiones que indiquen imposición, así como se evidencia en el enunciado "Pues como yo desde chiquita mi mamá me crio como hacer oficio en la casa", el pronombre "yo" indica que se refiere a que la acción recae solamente sobre la hija y es la mamá quien enseña instrucciones específicas de cómo hacer un oficio en un lugar. Además, en la expresión "pues como yo desde chiquita", la palabra "desde" es una preposición que indica origen o procedencia de tiempo, se genera una comparación en relación al momento pasado, "desde chiquita" con el momento de la narración en el cual la mujer ya es adulta; se establece una relación entre "chiquita" y "grande" mediada por el tiempo. Con el enunciado "usted va a ser grande usted desde chiquita" en la cual el padre le sentencia a su hija que durante toda su vida realizará los mismos oficios y labores, de esta manera se enseña a la niña que desde muy pequeña debe actuar como adulta. 
"¿Para qué vas a tener marido si vas a ser perezosa?"

Pues yo ya sabía todo, pues tenía la experiencia de así de adulta.

Filda Gómez Ahué, madre ticuna

Todos los oficios y labores que el padre y la madre imponen, enseñan y sentencian están encaminados a la vida adulta y al hecho de constituir familia. Es así como, siendo apenas una niña, debe obligarse a interiorizar todas las labores propias de una mujer adulta, de lo contrario no será posible que consiga una pareja. Tal como se expresa en el enunciado "ipara qué vas a tener marido si vas a ser perezosa?", la interrogación "¿para qué?" expresa la realización de una acción en función de "tener marido" lo cual se hace complicado de lograr sin haber aprendido antes determinadas labores; y la expresión condicional "si vas a ser perezosa" es muestra del desconocimiento de las labores, con lo cual no será posible conseguir una pareja. Por lo tanto, en el enunciado "pues yo ya sabía todo, pues tenía la experiencia de así de adulta", el pronombre "yo" acompañado del verbo en pasado "sabía", implica el aprendizaje de una acción y la expresión "pues tenía la experiencia" denota que siendo niña ya conocía y sabía hacer las labores que debía desempeñar de adulta.

Nacimiento, cuidados y creencias

Uno se va aparte... bien aparte estilo animal nadie tiene que ver nada.

Lo tenemos sentada de cuclillas y abrir la puerta y así es que sale.

Uno le hace morder uno mismo y ahí está curando el niño para que no sea hablador.

Alba lucía Laulate, abuela ticuna

Se indican una serie de acciones señaladas en la expresión "uno se va aparte... bien aparte estilo

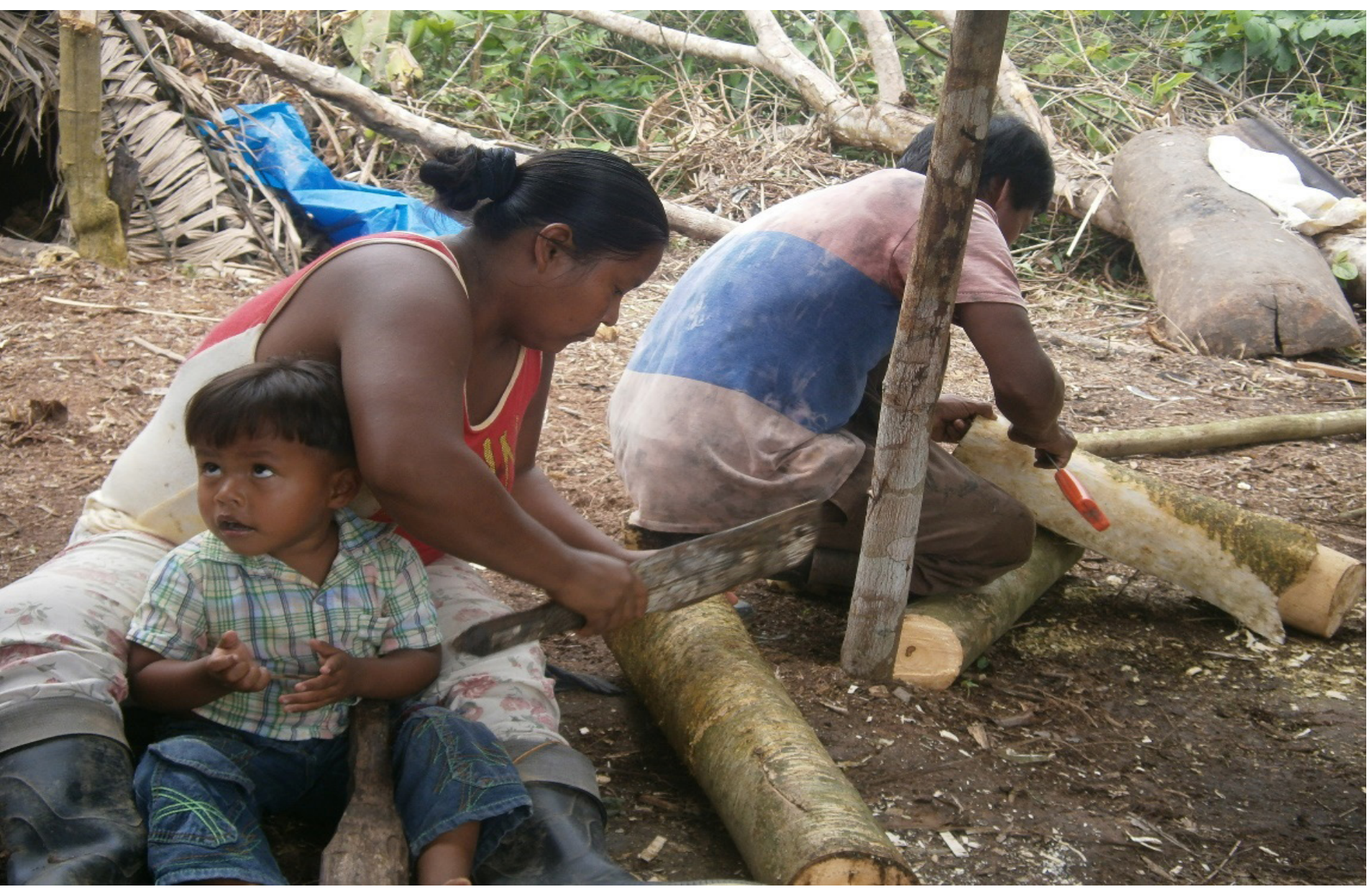

Figura 2. extracción de Yanchama en la chagra. Fuente: archivo fotográfico de la investigación. 
animal", en la reiteración de "bien aparte" demuestra una acción de movimiento hacia un lugar lejano que sugiere ser solitario. Se evidencia una analogía en la acción de parir de una mujer y de un animal sustentada en el hecho de tener que trasladarse. Además, se convierte en un suceso oculto, cuando la narradora indica que "nadie tiene que ver nada" esta expresión sugiere que la comunidad deberá alejarse del nacimiento ya que se mantiene como un acto secreto e íntimo solamente "la mamá y la partera" son quienes acompañan y reciben al recién nacido.

En cuanto a los saberes durante el parto, la madre relata "lo tenemos sentada de cuclillas y abrir la puerta y así es que sale", en el verbo en segunda persona del plural "lo tenemos" señala una acción compartida por el género femenino en un mismo contexto, en el cual se adopta una posición específica "sentada de cuclillas" que refiere la preparación del cuerpo para facilitar el nacimiento. En consecuencia, la madre manifiesta metafóricamen188 te "abrir la puerta y así es que sale" muestra el cuerpo de la madre como la casa que abre su puerta para recibir al recién nacido en el exterior.

La partera también a veces debe hacer de madrina.

Es ella quien entierra la placenta,

pero no muy hondo porque entre más hondo la entierra aja,

así serán las raíces de los dientes.

Alba lucía Laulate, abuela ticuna

La creencia contemplada desde el momento que se realizan las contracciones para recibir al niño y que tiene repercusión en el futuro está expresada en el enunciado "uno le hace morder uno mismo y ahí está curando el niño para que no sea hablador". En la expresión "uno le hace morder uno mismo" la madre reconoce la necesidad de morderse los labios con el fin de que "el niño... no sea hablador" esto es considerado como un símbolo particular de su creencia donde existe una relación directa entre los labios de la madre como instrumento de la palabra y el apropiado uso de la voz que la madre pretende para su hijo. La anterior expresión se muestra como una figura alegórica donde la idea figurativa es "no ser hablador" y el objeto concreto son "los labios mordidos por la mujer". En la comunidad indígena Ticuna la palabra tiene una función importante para la transmisión de la cultura, por esto desde el nacimiento la madre considera importante cultivar el buen uso de oralidad.

En el nacimiento, la partera tiene doble función: de médico y madrina. Es ella quien le pone el nombre al recién nacido. La importancia de este acto como lo plantea Goulard (p. 137, 2009) radica en que "la atribución de un nombre al recién nacido, es su verdadera inscripción en el mundo como ser vivo", y "es ella quien entierra la placenta, pero no muy hondo porque entre más hondo la entierra aja, así serán las raíces de los dientes", se evidencia en la narración una figura metafórica que compara el crecimiento de los dientes con la acción de enterrar la placenta. La figura expone una relación existente entre lo físico de la tierra y el devenir en el crecimiento de los dientes; el "enterrar" como acción de sepultar, de devolver a la tierra ese residuo del nacimiento. En la expresión "entre más hondo la entierra aja, así serán las raíces de los dientes" se crea una relación con el estado en el cual se prolonga la raíz de los dientes de acuerdo a la profundidad en que se entierre.

\section{Dieta y enfermedades}

El susto, mal aire, un choque de aire.

Nosotros las creencias de nosotros dice que hay veces viene un espíritu malo y le acaricia al niño.

Aliria Gómez, madre ticuna

Solo en la infancia se presenta un desequilibrio energético. En la expresión "el susto, mal aire, un choque de aire" refiere que la causa del susto es un viento que al colisionar con el niño causa una reacción negativa; que seguido de la frase "un aire malo, viene a ser así, así como ese ventarrón, uno no sale" se comprende en la expresión "viene a ser así" como método de la oralidad para hacerse entender en otras palabras, a quien no está cercano a sus saberes, usando una analogía con una experiencia similar.

En el enunciado "nosotros las creencias de nosotros dice que hay veces, viene un espíritu malo 
y le acaricia al niño", el pronombre "nosotros" cumple la función de agrupar en el discurso a varios individuos que practican acciones particulares y por ende hay una distancia con los otros que no la comparten, en este caso el sustantivo "creencias" se refiere a las ideologías que reúne a un grupo de personas, en este caso la etnia Ticuna, quienes reconocen la existencia de determinados espíritus. Estos conocimientos son aprendidos generacionalmente y con el verbo en tercera persona "dice" se establece como un mandato que lo convierte en una verdad cultural. Este precepto señala que, en ocasiones, "viene un espíritu malo y le acaricia al niño", la relatora al emplear el verbo acariciar alude que a pesar de ser un espíritu malo el que roza al niño y le causa alguna enfermedad, no transgrede su cuerpo, sino lo toca sutilmente creando un malestar que no demuestra herida física.

Entonces uno tiene que buscar un señor que rece, que sepa rezar pa' uno mandar rezar los niños, después de eso, pues uno ya le humea con cacho, y eso también le quita

[...] si usted no le manda rezar no le sana.

Aliria Gómez, madre ticuna

Existen entre la comunidad saberes sobre la curación de las enfermedades que adquieren los niños. Estos han sido otorgados a personas que saben rezar, los cuales definen un procedimiento para curar la enfermedad, así como se muestra en el enunciado "entonces uno tiene que buscar un señor que rece, que sepa rezar pa uno mandar rezar los niños, después de eso, pues uno ya le humea con cacho, y eso también le quita" son generalmente los abuelos las personas que poseen los conocimientos espirituales de la naturaleza para tratar cualquier mal que desequilibra la dimensión corporal. En este sentido, las afecciones son causadas por una descompensación espiritual, dado que las enfermedades son síntomas de un encuentro negativo con una entidad intangible, de allí que el soplo dado por la palabra tenga una función de limpiar. En el enunciado "si usted no le manda rezar no le sana" se comprende como el único método para asegurar el bienestar del niño.
Ritos y mitos: la pelazón

Es que cuando a una le llega el primer tiempo, eso te vienen a ser conocer, cuando ya tienen lo once años, te vienen a explicar el momento, por ejemplo, mi finada mamá arrancaba yuca conmigo y ella empezaba a explicarme [...] ella me decía usted tiene que tener cuidado hija usted ya está grande [...]. Tu no vas a estar en esta edad siempre, usted va a crecer, los senos crecen, esos primeros senos hay que curarle para cuando llegue el tiempo, ni estén comiendo cosas para que le hagan daño, antes de llegar la edad de 13 o 14 años, usted ya va a mirar el primer tiempo [...] le bañan con el árbol de Ubo para sacarle la saladera que trae esa sangre maligna aja, luego esa agua también le echan en la cabeza pa' que ablande el cabello [...]. Esa sangre de la niña aja, es maligna como la del parto aja, entonces pues ellos le recogen bien, bien y lo tienen que sembrar pidiendo al espiritual, ellos le hacen sagradamente y nadie tiene que mirar eso [...] se corta el pelo porque yo volví a nacer, se cambia de pelo, así me hicieron.

Alba Lucía Laulate, madre ticuna

El ritual que define el fin de la infancia y el inicio de la etapa de reproducción es Ilamado por los Ticuna como la pelazón, la cual está marcada por la llegada del primer tiempo en las mujeres, tal como lo expresa la frase "Es que cuando a una le llega el primer tiempo, eso te vienen a ser conocer, cuando ya tienen lo once años, te vienen a explicar el momento, por ejemplo mi finada mamá arrancaba yuca conmigo y ella empezaba a explicarme", en las actividades diarias que realiza con su madres hay un previo consejo en donde se hace una advertencia del devenir, ya que la cultura le advierte un cambio en su cuerpo que la posibilita a quedar en embarazo, condición por la cual tendrá que cuidarse, "Ella me decía «usted tiene que tener cuidado hija usted ya está grande»". La niña atraviesa un período en donde debe afrontar cambios corporales considerados como signos que señalan el inicio del ritual de la pelazón para preparación de la niña en su transición a ser mujer. En la frase "Tu no vas a estar en esta edad siempre, usted va a crecer, los senos crecen, esos primeros senos hay que curarle para cuando llegue el tiempo, ni estén comiendo cosas para que le hagan daño, antes de llegar la edad de 13 o 14 años, usted ya va a mirar el primer tiempo", los senos son la primera característica de 
esa transformación y para los Ticuna es importante cuidar el estado de fragilidad en la que entra su cuerpo. Por ello se cura y se aísla para no ser vulnerada. Se infiere que ser mujer al interior de la comunidad es la adecuación del cuerpo para sostener el crecimiento de otra vida, por esto los senos son parte fundamental en el proceso de la maternidad, de allí que el cambio de la niña es una adecuación para ser mujer en un sentido biológico.

Llegado el momento en el cual la niña es encerrada, es decir, aislada y protegida en su estado de fragilidad, la familia hace sus preparativos para llevar a cabo el ritual. Entre los insumos necesarios para la protección de la niña se encuentran las plantas, las cuales poseen características espirituales y energía vital, así como lo explica (Goulard, 2009) con los sistemas de referencia compartida, en donde el ser humano existe a partir de tres principios o fuerzas:

Un principio corporal (ma-ü) que desaparece con la muerte, un principio vital (a-e) que se desarrolla progresivamente en el transcurso de la existencia del individuo, y una fuente de energía (porá) que lo mantiene vivo y debe ser constantemente realimentada. (Goulard, 2009, p 18)

Las plantas son fuente que realimenta el principio energético del ser humano, de allí que en el ritual de la pelazón se hace uso del piri-piri, como protección espiritual, el árbol de ubo que se enuncia en la frase "le bañan con el árbol de ubo para sacarle la saladera que trae esa sangre maligna aja, luego esa agua también le echan en la cabeza pa que ablande el cabello" interviene en el momento de la ceremonia con dos funciones una es evitar la mala fortuna a causa de la primera menstruación, sangre que se considera negativa puesto que el organismo la expulsa en forma de desecho para dar paso a una nueva etapa: "Esa sangre de la niña aja, es maligna como la del parto aja, entonces pues ellos le recogen bien, bien y lo tienen que sembrar pidiendo al espiritual, ellos le hacen sagradamente y nadie tiene que mirar eso". La sangre es llevada a la tierra y se siembra de manera solemne haciendo de esta acción un acto secreto. Dos, facilita la extracción del pelo, humedeciendo el cuero cabelludo con el agua del árbol de ubo, el cual es considerado el árbol de la vida, por tanto, se entiende el hecho de cortar el cabello para dar un nuevo inicio, en concordancia con la frase "se corta el pelo porque yo volví a nacer, se cambia de pelo, así me hicieron". Goulard plantea que la niña entra en un estado de adormecimiento donde el tiempo se establece diferente, con otra finalidad, regenera el cuerpo, mas no lo envejece, se trata del nacimiento de un ser completo que llega a la plenitud, es decir, a la edad adulta.

Las plantas son importantes como objetos que trascienden lo físico a lo simbólico. En ellas se puede tener la confianza de los antepasados que fueron gente y, así mismo, conocedores de las lógicas de la naturaleza, es allí en el ritual donde todo cobra sentido, la niña establece una conexión con la naturaleza que es el puente entre el mundo espiritual y físico otorgándole la fuerza para cumplir con la ceremonia de la pelazón.

La niña es encerrada porque se considera que entra en un estado liminal, donde la pueden acechar diferentes entidades del monte y enfermar, es por esto que no puede establecer contacto con nadie. La connotación que se le da al habla es importante puesto que el silencio debe estar presente en el ritual como símbolo de reflexión interna y la palabra debe ser reservada para evitar intenciones negativas.

\section{Conclusiones}

La investigación permitió acercarnos a las prácticas y saberes de crianza al interior de la comunidad ticuna, lo cual enriqueció el conocimiento frente a las realidades indígenas del departamento de Amazonas en Colombia, a partir de un intercambio de experiencias cotidianas con miembros de las familias que construyen sus dinámicas de vida en relación con su contexto social, además se logró reconocer la vital importancia de la oralidad para entender su cultura.

A través de la indagación, se puede evidenciar que posiblemente la identidad histórica de las familias entrevistadas de la comunidad indígena ticuna comprenden su lugar en el mundo a través de la cosmogonía mítica, la cual es una estructura 
ordenada y sistemática que da sentido a sus prácticas y rituales. En la actualidad, su existencia se transforma y confronta con múltiples culturas que subsisten en la tensión de la modernidad y lo tradicional. El método científico está latente en el sistema de las sociedades occidentales en una lógica racional que desacredita el pensamiento mítico de las sociedades denominadas primitivas. Mientras que las familias entrevistadas manifiestan en sus prácticas cotidianas que conviven en esta tensión sin acreditar un pensamiento que sea superior, aceptando que existen diferentes lenguajes para comprender el mundo.

Los rituales son ceremonias colectivas que contienen representaciones simbólicas basadas en el mito de origen para traer al presente la memoria ancestral, por ende, tiene un sentido sagrado al interior del pueblo ticuna que busca preservar su cultura. Un ritual destacado en la comunidad es la pelazón, el cual representa el cambio biológico de niña a mujer en contacto con la naturaleza y la espiritualidad; refleja a la mujer como un ser incompleto que transita hacia su plenitud definida como la posibilidad de concebir vida. Actualmente, este ritual persiste en la memoria de los abuelos sabedores y en la oralidad de la comunidad, no obstante, la práctica de esta ceremonia se ha transformado por la inmersión de doctrinas religiosas e institucionales.

La cultura indígena ticuna ha sido permeada por la presencia de los aparatos del Estado, los cuales no están adaptados a las realidades del territorio. La escuela aparece como una institución que irrumpe los lugares naturales de la educación propia, puesto que el sistema educativo escolar de carácter religioso aleja a los niños de su comunidad para internarlos y desarticularlos de sus costumbres natales, que aunque amplían sus horizontes de vida, pocos regresan para aportar los conocimientos aprendidos; los entes de salud pública cambian las tradiciones de curación y asistencia, por la apropiación de métodos asépticos promocionados para modificar algunas prácticas como el alumbramiento, la planificación familiar y el cuidado en las enfermedades, esto modifica la concepción de vida en los ticuna que pone de presente la espiritualidad y la intimidad.

Las prácticas de crianza fueron indagadas al interior de la comunidad a partir del análisis de relatos que dieron cuenta de la familia como primer escenario de aprendizaje en donde se legitiman comportamientos, prácticas y formas de sentir para entender el mundo. Cada integrante presenta una función educativa para la infancia, las abuelas, abuelos y madres representan la sabiduría ancestral que se transmite mediante el consejo, como el deber ser; mientras que la función del padre es la figura de ley, es decir el deber hacer; los niños y niñas por su parte son reconocidos como aprendices de la vida adulta, que se preparan mediante la observación y la práctica para prolongar la familia, principal objetivo de las dinámicas de vida en la comunidad.

Este estilo de vida es considerado por otras sociedades mayoritarias como índice de pobreza, puesto que el aumento de natalidad es una condición que deteriora la calidad de vida en sus habitantes. Sin embargo, el plan de vida en los indígenas está atravesado por construcciones culturales propias que dan importancia a otras categorías de bienestar que implican la supervivencia y aumento de la población.

En cuanto a la crianza, los relatos arrojaron temáticas de discusión que posibilitaron un análisis para comprender el conjunto de sus prácticas como lo son: preparación de saberes y oficios para la unión de pareja en donde se destaca que en las 
actividades cotidianas que debe aprender una mujer ticuna, el hacer es el resultado de lo aprendido de padre y madre, que pasa por la observación, comprensión e interiorización de un saber que posteriormente es llevado a cabo durante toda la vida.

En los enunciados concernientes a nacimiento, cuidados y creencias; y dieta y enfermedades, se encontró que existen conocimientos y saberes que prevalecen para cuidar la vida, como el cuidado con la naturaleza y los espíritus que habitan en ella para obtener beneficios mutuos, el cuidado con el recién nacido quien adquiere una responsabilidad con la comunidad basada en la continuidad de las costumbres que son realizadas por el cuidador y observadas por el niño dando origen a sus hábitos de vida. Al momento de nacer, el bebé es anidado en el tejido cultural de la comunidad, la cual concibe la existencia como un tiempo que transcurre en un sentido cíclico, puesto que la muerte no acaba con la vida, la transforma en entidades espirituales que intervienen en el crecimiento adecuado tenien-

192 do en cuenta el desarrollo corporal y el cuidado de su ser.

El uso de la lengua materna está latente en las familias entrevistadas. Es importante señalar que existe una conexión afectiva al momento de conversar en idioma ya que es más usual escucharlos hablar en espacios familiares, que creen un ambiente íntimo y de confianza. Sin embargo, ya no todos los pobladores hablan la lengua (aunque la entienden) y el español es usado como herramienta de comunicación adaptado a las estructuras del pensamiento indígena, es decir, es un español que se ha modificado a las características culturales propias.

En este sentido, el discurso empleado por algunos de los habitantes de la comunidad Doce de octubre posee el uso de figuras lingüísticas elaboradas con abundancia metafórica, característico de su oralidad. A partir de la metáfora se crean vínculos simbólicos entre el hacer y el pensar propios de su cosmogonía. La palabra hablada se convierte en una herramienta para subsistir a la temporalidad pues la narración es compartida generacionalmente. Los abuelos, principales actores para la permanencia de la cultura, hablan de su experiencia como hecho sagrado dado que está unido a la concepción mitológica de origen, el cual permea las funciones del hombre e ideas morales sobre el acto de existir que luego las nuevas generaciones apropian como el código narrativo cultural, de allí que la metáfora aparezca como el medio para interpretar las realidades vivenciadas y resignificadas por la descendencia familiar ticuna.

Esta comunidad construye su relato asumiendo su razón de ser como parte de la naturaleza, es decir, "un ser vivo más" contrario a otras concepciones en donde se intenta dominarla. Por lo tanto, para sus habitantes, el contexto tiene voz y dialoga con sus distintas especies; la oralidad entonces no está sujeta solamente a la palabra, también a las acciones. Existe un lenguaje de la naturaleza que los ticuna escuchan y de allí aprenden, por ejemplo, el conocimiento sobre el devenir de la sangre expulsada por la mujer en su primera menstruación, la cual es considerada maligna es una interpretación simbólica de la comunicación con la naturaleza que guía a la comunidad ante distintos acontecimientos del ciclo vital en el ser humano.

Respecto a la oralidad, la palabra no se desprende del sujeto que la evoca, porque allí es donde tiene vida, ejercicio diferente en la palabra escrita la cual toma prestada la voz de quien la lee. En la oralidad, las palabras tienen personalidad, asumen la expresión gestual del emisor, la tonalidad sonora y el ánimo del relator, si se quiere es una experiencia única que está estrechamente relacionada con el espacio, lo significa y transforma, hasta volverse contexto, mientras que en los sistemas de escritura el lenguaje hace un pacto con el tiempo. Los cambios en la historia de la humanidad surgen a partir de la escritura, la cual se erige como la historia oficial, su objetivo es permanecer en el tiempo hacer perenne un hecho.

Tanto la escritura como la oralidad son necesarias, una dispuesta a la otra, no obstante, aquí se intenta ahondar en las características de la oralidad, la riqueza del relato como narración natural del hombre dado en la cultura. Las narraciones orales son una característica constante de la mayoría de comunidades nativas, en donde vinculan su existencia a parámetros míticos ancestrales.

En lo particular de los ticuna, o bien en los relatos de origen mitológico narrados por los pobladores se comprende la aceptación de la muerte, el 
reconocimiento de un sistema cíclico natural por el que transitan todas las especies. Esto se comprende, por ejemplo, cuando en sus relatos, ciertos animales personifican un grado alto de sabiduría que fue obtenida a lo largo de su existencia en la historia mitológica y que aparece como símbolo ante las distintas generaciones con un legado protector.

De lo anterior podríamos deducir que la oralidad es una condición de narrar la historia sujeta al contexto, que cambia y se transforma en la medida que es interpretada por próximas generaciones pero que conserva un sentido mítico. La oralidad está sujeta al espacio y narra la vida. Se podría comprender a la oralidad como una tinaja que transporta la sabiduría por todas las voces que visita.

Para terminar, esta investigación abre paso a nuevas indagaciones que permita profundizar en la oralidad ticuna, que explore nuevos campos y por ende salga de los lugares comunes. La metáfora aparece como un elemento trascendental en la oralidad de la comunidad Doce de octubre, dado que sobrepasa el carácter decorativo sobre el relato, más bien es una facultad que desarrolla ingenio para narrar el acontecimiento que pasa por el hecho descriptivo para realizar una interpretación simbólica de la experiencia, que al pasar por el sentir permite configurar lo efímero en una representación perenne. Las palabras son el refugio del acto que crean un puente de relatos donde se encuentran las experiencias y permiten sostener con vida un tiempo de la contingencia del presente que perdura en la memoria de la voz, como si esta tuviera fibras de yanchama que visten la cultura de una lengua materna viva.

\section{Referencias}

Bruner, J. (1991). Actos de significado: más allá de la revolución cognitiva. Madrid: Alianza.

Cabrejo, E. (2007). El relato como fuente de organización y creación infantil. En: F. A. Santamaría; M. H. Barreto, Lenguaje y saberes infantiles (pp. 75-91). Bogotá: Universidad Distrital Francisco José de Caldas.

Canclini, N. (2012). El horizonte ampliado de la interculturalidad. Hibridación e interculturalidad. México: Clacso.

Fajardo, G. (1986). Visión etnográfica de los Ticuna de San Martín de Amacayacu. Bogotá: Departamento de Antropología, Facultad de Ciencias Humanas, Universidad Nacional de Colombia.

Garfinkel, H. (2006). Estudios en Etnometodología. Barcelona: Anthropos.

Goulard, J. P. (2009). Entre mortales e inmortales. El Ser según los ticuna de la Amazonía. Lima: CAAAP/IFEA.

Guber, R. (2001). La etnografía. Método, campo y reflexividad. Bogotá: Grupo Editorial Norma.

Landaburu, J. R. (1984). Tradiciones de la gente del hacha: mitología de los indios andoques del Amazonas. Bogotá: Yerbabuena: Instituto Caro y Cuervo/Unesco.

Mondada, L. (2006). Espacio y lenguaje. En: D. Hiernaux, Tratado de geografía humana (pp. 12-33). México: Universidad Autónoma de México. 